\title{
ON THE DEPTH OF SYMBOLIC POWERS OF EDGE IDEALS OF GRAPHS
}

\author{
S. A. SEYED FAKHARI
}

\begin{abstract}
Assume that $G$ is a graph with edge ideal $I(G)$ and star packing number $\alpha_{2}(G)$. We denote the $s$-th symbolic power of $I(G)$ by $I(G)^{(s)}$. It is shown that the inequality depth $S /\left(I(G)^{(s)}\right) \geq \alpha_{2}(G)-s+1$ is true for every chordal graph $G$ and every integer $s \geq 1$. Moreover, it is proved that for any graph $G$, we have $\operatorname{depth} S /\left(I(G)^{(2)}\right) \geq \alpha_{2}(G)-1$.
\end{abstract}

\section{INTRODUCTION}

Let $\mathbb{K}$ be a field and $S=\mathbb{K}\left[x_{1}, \ldots, x_{n}\right]$ be the polynomial ring in $n$ variables over $\mathbb{K}$. Computing and finding bounds for the depth (or equivalently, projective dimension) of homogenous ideals of $S$ and their powers have been studied by several authors (see e.g., [3], 4], [5], 8], [9], [1], [12], [13]).

In [7, Fouli, Hà and Morey introduced the notion of initially regular sequence. Using this notion, they provided a method for estimating the depth of a homogenous ideal. To be more precise, let $I \subset S$ be a homogenous ideal and let $\left\{b_{i, j} \mid 1 \leq i \leq q, 0 \leq\right.$ $\left.j \leq t_{i}\right\}$ be a subset of distinct variables of $S$. Suppose $\operatorname{in}_{<}(\mathrm{I})$ is the initial ideal of $I$ with respect to a fixed monomial order $<$ and assume that $G\left(\operatorname{in}_{<}(I)\right)=\left\{u_{1}, \ldots, u_{m}\right\}$ is the set of minimal monomial generators of $\mathrm{in}_{<}(I)$. It is shown in [7, Theorem 3.11] that depth $S / I \geq q$, provided that the following conditions hold.

(i) The monomials $u_{1}, u_{2}, \ldots, u_{m}$ are not divisible by $b_{i, j}^{2}$ for $1 \leq i \leq q$ and $1 \leq j \leq t_{i}$.

(ii) For $i=1,2, \ldots, q$, if a monomial in $\left\{u_{1}, \ldots, u_{m}\right\}$ is divisible by $b_{i, 0}$, then it is also divisible by $b_{i, j}$, for some integer $1 \leq j \leq t_{i}$,

In Section 2, we provide an alternative proof for this result (see Proposition 2.11). Our proof is based on a short exact sequence argument, while in [7], the authors construct an initially regular sequence to prove their result.

Fouli, Hà and Morey [6] observed that the above result provides a combinatorial lower bound for the depth of edge ideals of graphs. Indeed, for every graph $G$ with edge ideal $I(G)$, we have

$$
\operatorname{depth} S / I(G) \geq \alpha_{2}(G),
$$

2000 Mathematics Subject Classification. Primary: 13C15, 13F55, 05E40.

Key words and phrases. Chordal graphs, Depth, Edge ideal, Star packing number, Symbolic powers. 
where $\alpha_{2}(G)$ denotes the so-called star packing number of $G$ (see Section 2 for the definition of star packing number and see Corollary 2.2 for more details about the above inequality). It is proven in [6, Theorem 3.7] that the above inequality can be extended to powers of $I(G)$ when $G$ is a forest. More precisely, for every forest $G$ and for every integer $s \geq 1$, the inequality

$$
\operatorname{depth} S / I(G)^{s} \geq \alpha_{2}(G)-s+1
$$

holds. On the other hand, we know from [16, Theorem 5.9] that for every forest $G$, the $s$-th ordinary and symbolic powers of $I(G)$ coincide. Hence, inequality $(\oplus$ ) essentially says that for every forest $G$ and any positive integer $s$,

$$
\operatorname{depth} S / I(G)^{(s)} \geq \alpha_{2}(G)-s+1 \text {. }
$$

In Theorem 3.4, we generalize [6, Theorem 3.7] by proving inequality (\$) for any chordal graph. Moreover, we show that inequality $(\$)$ is true for $s=2$ and for any graph $G$ (see Theorem 4.2).

\section{Preliminaries AND KNOWN RESUlts}

In this section, we provide the definitions and the known results which will be used in the next sections.

Let $G$ be a simple graph with vertex set $V(G)=\left\{x_{1}, \ldots, x_{n}\right\}$ and edge set $E(G)$. For a vertex $x_{i}$, the neighbor set of $x_{i}$ is $N_{G}\left(x_{i}\right)=\left\{x_{j} \mid x_{i} x_{j} \in E(G)\right\}$. We set $N_{G}\left[x_{i}\right]=N_{G}\left(x_{i}\right) \cup\left\{x_{i}\right\}$ and call it the closed neighborhood of $x_{i}$. The cardinality of $N_{G}\left(x_{i}\right)$ is the degree of $x_{i}$ and will be denoted by $\operatorname{deg}_{G}\left(x_{i}\right)$. For every subset $U \subset V(G)$, the graph $G \backslash U$ has vertex set $V(G \backslash U)=V(G) \backslash U$ and edge set $E(G \backslash U)=\{e \in E(G) \mid e \cap U=\emptyset\}$. A subgraph $H$ of $G$ is called induced provided that two vertices of $H$ are adjacent if and only if they are adjacent in $G$. A graph $G$ is called chordal if it has no induced cycle of length at least four. A subset $W$ of $V(G)$ is a clique of $G$ if every two distinct vertices of $W$ are adjacent in $G$. A vertex $x$ of $G$ is a simplicial vertex if $N_{G}(x)$ is a clique. It is well-known that every chordal graph has a simplicial vertex. A subset $C$ of $V(G)$ is a vertex cover of $G$ if every edge of $G$ is incident to at least one vertex of $C$. A vertex cover $C$ is a minimal vertex cover if no proper subset of $C$ is a vertex cover of $G$. The set of minimal vertex covers of $G$ will be denoted by $\mathcal{C}(G)$. A subset $A$ of $V(G)$ is called an independent subset of $G$ if there are no edges among the vertices of $A$. Obviously, $A$ is independent if and only if $V(G) \backslash A$ is a vertex cover of $G$.

The edge ideal of a graph $G$ is defined as

$$
I(G)=\left(x_{i} x_{j} \mid x_{i} x_{j} \in E(G)\right) .
$$

For a subset $C$ of $\left\{x_{1}, \ldots, x_{n}\right\}$, we denote by $\mathfrak{p}_{C}$, the monomial prime ideal which is generated by the variables belonging to $C$. It is well-known that for every graph $G$,

$$
I(G)=\bigcap_{C \in \mathcal{C}(G)} \mathfrak{p}_{C}
$$


Let $I$ be an ideal of $S$ and let $\operatorname{Min}(I)$ denote the set of minimal primes of $I$. For every integer $s \geq 1$, the $s$-th symbolic power of $I$, denoted by $I^{(s)}$, is defined to be

$$
I^{(s)}=\bigcap_{\mathfrak{p} \in \operatorname{Min}(I)} \operatorname{Ker}\left(S \rightarrow\left(S / I^{s}\right)_{\mathfrak{p}}\right) .
$$

Let $I$ be a squarefree monomial ideal in $S$ and suppose that $I$ has the irredundant primary decomposition

$$
I=\mathfrak{p}_{1} \cap \ldots \cap \mathfrak{p}_{r},
$$

where every $\mathfrak{p}_{i}$ is an ideal generated by a subset of the variables of $S$. It follows from [10, Proposition 1.4.4] that for every integer $s \geq 1$,

$$
I^{(s)}=\mathfrak{p}_{1}^{s} \cap \ldots \cap \mathfrak{p}_{r}^{s} .
$$

We set $I^{(s)}=S$, for any integer $s \leq 0$.

It is clear that for any graph $G$ and every integer $s \geq 1$,

$$
I(G)^{(s)}=\bigcap_{C \in \mathcal{C}(G)} \mathfrak{p}_{C}^{s}
$$

As it was mentioned in introduction, Fouli, Hà and Morey [7] detected a method to bound the depth of a homogenous ideal. We provide an alternative proof for their result. Recall that for every monomial $u$ and for every variable $x_{i}$, the degree of $u$ with respect to $x_{i}$ is denoted by $\operatorname{deg}_{x_{i}}(u)$.

Proposition 2.1 ([7], Theorem 3.11). Let I be a proper homogenous ideal of $S$ and let $<$ be a monomial order. Assume that $B=\left\{b_{i, j} \mid 1 \leq i \leq q, 0 \leq j \leq t_{i}\right\}$ is a subset of distinct variables of $S$, such that the following conditions are satisfied.

(i) For every pair of integers $1 \leq i \leq q, 1 \leq j \leq t_{i}$ and for every $u \in G\left(\operatorname{in}_{<}(I)\right)$, we have $\operatorname{deg}_{b_{i, j}}(u) \leq 1$.

(ii) For $i=1,2, \ldots, q$, if a monomial $u \in G\left(\operatorname{in}_{<}(I)\right)$ is divisible by $b_{i, 0}$, then it is also divisible by $b_{i, j}$, for some integer $1 \leq j \leq t_{i}$.

Then depth $S / I \geq q$.

Proof. It is known that depth $S / I \geq \operatorname{depth} S / \operatorname{in}_{<}(I)$ (see e.g., [10, Theorem 3.3.4]). Hence, replacing $I$ by $\operatorname{in}_{<}(I)$, we may suppose that $I$ is a monomial ideal. We use induction on $|B|$. There is nothing to prove for $|B|=0$, as in this case $q=0$. Therefore, assume that $|B| \geq 1$. If $t_{i}=0$, for every $i=1,2, \ldots, q$, then it follows from condition (ii) that $b_{1,0}, \ldots, b_{q, 0}$ do not divide the minimal monomial generators of $I$. In particular, they form a regular sequences on $S / I$ and the assertion follows. Thus, suppose that $t_{i} \geq 1$, for some $i$ with $1 \leq i \leq q$. Without lose of generality, suppose $i=1$. Consider the following short exact sequence.

$$
0 \longrightarrow S /\left(I: b_{1, t_{1}}\right) \longrightarrow S / I \longrightarrow S /\left(I, b_{1, t_{1}}\right) \longrightarrow 0
$$

This yields that

$$
\operatorname{depth} S / I \geq \min \left\{\operatorname{depth} S /\left(I: b_{1, t_{1}}\right), \operatorname{depth} S /\left(I, b_{1, t_{1}}\right)\right\} \text {. }
$$


By condition (i), the variable $b_{1, t_{1}}$ does not appear in the minimal monomial generators of $\left(I: b_{1, t_{1}}\right)$. In particular, $b_{1, t_{1}}$ is a regular element on $S /\left(I: b_{1, t_{1}}\right)$. Let $S^{\prime}$ be the polynomial ring obtained from $S$ by deleting the variable $b_{1, t_{1}}$ (in other words, $\left.S^{\prime} \cong S /\left(b_{1, t_{1}}\right)\right)$. Set $I^{\prime}:=\left(I: b_{1, t_{1}}\right) \cap S^{\prime}$. It follows that

$$
\operatorname{depth} S /\left(I: b_{1, t_{1}}\right)=\operatorname{depth} S /\left(\left(I: b_{1, t_{1}}\right), b_{1, t_{1}}\right)+1=\operatorname{depth} S^{\prime} / I^{\prime}+1 .
$$

Clearly, $I^{\prime}$ satisfies the assumptions with respect to the set $\left\{b_{i, j} \mid 2 \leq i \leq q, 0 \leq j \leq t_{i}\right\}$ of variables. Thus, the induction hypothesis implies that depth $S^{\prime} / I^{\prime} \geq q-1$. Hence, we deduce from the above equalities that

$$
\operatorname{depth} S /\left(I: b_{1, t_{1}}\right) \geq q \text {. }
$$

Using inequality (11), it suffices to prove that depth $S /\left(I, b_{1, t_{1}}\right) \geq q$. Set $I^{\prime \prime}:=I \cap S^{\prime}$. Then $S /\left(I, b_{1, t_{1}}\right) \cong S^{\prime} / I^{\prime \prime}$. Put $t_{1}^{\prime}:=t_{1}-1$ and $t_{i}^{\prime}:=t_{i}$, for $i=2, \ldots, q$. Obviously, $I^{\prime \prime}$ satisfies the assumptions with respect to the set $\left\{b_{i, j} \mid 1 \leq i \leq q, 0 \leq j \leq t_{i}^{\prime}\right\}$ of variables. Therefore, we conclude from the induction hypothesis that

$$
\operatorname{depth} S /\left(I, b_{1, t_{1}}\right)=\operatorname{depth} S^{\prime} / I^{\prime \prime} \geq q \text {. }
$$

Let $G$ be a graph and $x$ be a vertex of $G$. The subgraph $\operatorname{St}(x)$ of $G$ with vertex set $N_{G}[x]$ and edge set $\left\{x y \mid y \in N_{G}(x)\right\}$ is called a star with center $x$. A star packing of $G$ is a family $S$ of stars in $G$ which are pairwise disjoint, i.e., $V(\operatorname{St}(x)) \cap V\left(\operatorname{St}\left(x^{\prime}\right)\right)=\emptyset$, for $\operatorname{St}(x), \operatorname{St}\left(x^{\prime}\right) \in S$. The quantity

$$
\max \{|S| \mid S \text { is a star packing of } G\}
$$

is called the star packing number of $G$. Following [6], we denote the star packing number of $G$ by $\alpha_{2}(G)$.

The following corollary is an immediate consequence of Proposition 2.1, and it was indeed observed in [6].

Corollary $2.2([6])$. For every graph $G$, we have

$$
\operatorname{depth} S / I(G) \geq \alpha_{2}(G) \text {. }
$$

Proof. Let $b_{1,0}, \ldots, b_{q, 0}$ be the centers of stars in a largest star packing of $G$. Moreover, for $1 \leq i \leq q$, assume that $N_{G}\left(b_{i, 0}\right)=\left\{b_{i, 1}, \ldots, b_{i, t_{i}}\right\}$. Then the assumptions of Proposition 2.1 are satisfied and it follows that

$$
\operatorname{depth} S / I(G) \geq q=\alpha_{2}(G) \text {. }
$$

\section{Symbolic POWERS OF EDGE IDEALS OF CHORDAL GRAPHS}

In this section, we prove the first main result of this paper, Theorem 3.4 which states that inequality $\boxplus$ is true for every chordal graph $G$ and for any integer $s \geq 1$. In order to prove this result, we first need to estimate the star packing number of the 
graph obtained from $G$ by deleting a certain subset of its vertices. This will be done in the following two lemmas.

Lemma 3.1. Let $G$ be a graph and let $W$ be a subset of $V(G)$. Then for every $A \subseteq \bigcup_{x \in W} N_{G}[x]$, we have

$$
\alpha_{2}(G \backslash A) \geq \alpha_{2}(G)-|W| .
$$

Proof. Let $\mathcal{S}$ be the set of the centers of stars in a largest star packing of $G$. In particular, $|\mathcal{S}|=\alpha_{2}(G)$. Since every vertex in $A$ belongs to the closed neighborhood of a vertex in $W$, it follows from the definition of star packing that $|\mathcal{S} \cap A| \leq|W|$. Then the stars in $G \backslash A$ centered at the vertices in $\mathcal{S} \backslash A$ form a star packing in $G \backslash A$ of size at least $\alpha_{2}(G)-|W|$. Therefore, $\alpha_{2}(G \backslash A) \geq \alpha_{2}(G)-|W|$.

Lemma 3.2. Assume that $G$ is a graph and $W=\left\{x_{1}, \ldots, x_{d}\right\}$ is a clique of $G$. Let $A$ be a subset of $V(G)$ such that

(i) $A \subseteq \bigcup_{i=1}^{d} N_{G}\left(x_{i}\right)$,

(ii) $N_{G}\left(x_{1}\right) \backslash\left\{x_{2}, \ldots, x_{d}\right\} \subseteq A$, and

(iii) $x_{1} \notin A$.

Then $\alpha_{2}(G \backslash A) \geq \alpha_{2}(G)-d+1$.

Proof. Let $\mathcal{S}$ be the set of the centers of stars in a largest star packing of $G$. Similar to the proof of the Lemma [3.1. we have $|\mathcal{S} \cap A| \leq d$. If $|\mathcal{S} \cap A| \leq d-1$, then the stars in $G \backslash A$ centered at the vertices in $\mathcal{S} \backslash A$ form a star packing in $G \backslash A$ of size at least $\alpha_{2}(G)-d+1$. Thus, the assertion follows in this case. Therefore, suppose $|\mathcal{S} \cap A|=d$. In this case, we have

$$
x_{1}, \ldots, x_{d} \in \bigcup_{x \in \mathcal{S} \cap A} N_{G}(x) .
$$

It again follows from the definition of star packing that

$$
x_{1}, \ldots, x_{d} \notin \bigcup_{x \in \mathcal{S} \backslash A} N_{G \backslash A}(x) .
$$

Therefore, we conclude from condition (ii) that

$$
N_{G \backslash A}\left[x_{1}\right] \cap\left(\bigcup_{x \in \mathcal{S} \backslash A} N_{G \backslash A}(x)\right) \subseteq\left\{x_{1}, \ldots, x_{d}\right\} \cap\left(\bigcup_{x \in \mathcal{S} \backslash A} N_{G \backslash A}(x)\right)=\emptyset .
$$

As a consequence, the stars in $G \backslash A$ centered at the vertices in $(\mathcal{S} \backslash A) \cup\left\{x_{1}\right\}$ form a star packing in $G \backslash A$ of size $\alpha_{2}(G)-d+1$. This completes the proof of the lemma.

We are now ready to prove that inequality $\ddagger$ holds for any chordal graph. Indeed, we are able to prove the following stronger result.

Proposition 3.3. Let $G$ be a chordal graph. Suppose $H$ and $H^{\prime}$ are subgraphs of $G$ with

$$
E(H) \cap E\left(H^{\prime}\right)=\emptyset \quad \text { and } \quad E(H) \cup E\left(H^{\prime}\right)=E(G) .
$$


Assume further that $H$ is a chordal graph. Then for every integer $s \geq 1$,

$$
\operatorname{depth} S /\left(I(H)^{(s)}+I\left(H^{\prime}\right)\right) \geq \alpha_{2}(G)-s+1 .
$$

Proof. As the isolated vertices have no effect on edge ideals, we assume that $V(H)=$ $V\left(H^{\prime}\right)=V(G)$ (i.e., we extend the vertex sets of $H$ and $H^{\prime}$ to $V(G)$ ). We use induction on $s+|E(H)|$. For $s=1$, we have $I(H)^{(s)}+I\left(H^{\prime}\right)=I(G)$ and the assertion follows from Corollary 2.2. Therefore, suppose $s \geq 2$. If $E(H)=\emptyset$, then $I\left(H^{\prime}\right)=I(G)$ and again we have the required inequality by Corollary 2.2 . Hence, we assume $|E(H)| \geq 1$.

To simplify the notations, we set $I:=I(H)^{(s)}+I\left(H^{\prime}\right)$. Since $H$ is a chordal graph, it has a simplicial vertex, say $x_{1}$, with nonzero degree. Without loss of generality, suppose $N_{H}\left(x_{1}\right)=\left\{x_{2}, \ldots, x_{d}\right\}$, for some integer $d \geq 2$. Consider the following short exact sequence.

$$
0 \longrightarrow \frac{S}{\left(I: x_{1} \ldots x_{d}\right)} \longrightarrow \frac{S}{I} \longrightarrow \frac{S}{\left(I, x_{1} \ldots x_{d}\right)} \longrightarrow 0
$$

Using depth Lemma [2, Proposition 1.2.9], we have

$$
\operatorname{depth} S / I \geq \min \left\{\operatorname{depth} S /\left(I: x_{1} \ldots x_{d}\right), \operatorname{depth} S /\left(I, x_{1} \ldots x_{d}\right)\right\} .
$$

By assumption, for every pair of integers $i \neq j$, with $1 \leq i, j \leq d$ we have $x_{i} x_{j} \in$ $E(H)$. Therefore, $x_{i} x_{j}$ is not an edge of $H^{\prime}$. Set

$$
U:=\bigcup_{i=1}^{d} N_{H^{\prime}}\left[x_{i}\right]
$$

and

$$
U^{\prime}:=\bigcup_{i=1}^{d} N_{H^{\prime}}\left(x_{i}\right) .
$$

Then using [14, Lemma 2], we have

$$
\begin{aligned}
& \left(I: x_{1} \ldots x_{d}\right)=\left(\left(I(H)^{(s)}+I\left(H^{\prime}\right)\right): x_{1} \ldots x_{d}\right) \\
& =I(H)^{(s-d+1)}+I\left(H^{\prime} \backslash U\right)+\left(\text { the ideal generated by } U^{\prime}\right) \\
& =I\left(H \backslash U^{\prime}\right)^{(s-d+1)}+I\left(H^{\prime} \backslash U\right)+\left(\text { the ideal generated by } U^{\prime}\right) .
\end{aligned}
$$

This yields that

$$
\operatorname{depth} S /\left(I: x_{1} \ldots x_{d}\right)=\operatorname{depth} S^{\prime} /\left(I\left(H \backslash U^{\prime}\right)^{(s-d+1)}+I\left(H^{\prime} \backslash U\right)\right),
$$

where $S^{\prime}=\mathbb{K}\left[x_{i}: 1 \leq i \leq n, i \notin U^{\prime}\right]$. Let $G^{\prime}$ be the union of $H \backslash U^{\prime}$ and $H^{\prime} \backslash U$. In fact, $G^{\prime}$ is the induced subgraph of $G$ on $V(G) \backslash U^{\prime}$. Clearly, $N_{G}\left(x_{1}\right) \backslash\left\{x_{2}, \ldots, x_{d}\right\}$ is contained in $U^{\prime}$. Then the above equality together with Lemma 3.2 and the induction hypothesis implies that

$$
\operatorname{depth} S /\left(I: x_{1} \ldots x_{d}\right) \geq \alpha_{2}\left(G \backslash U^{\prime}\right)-(s-d+1)+1 \geq \alpha_{2}(G)-s+1 .
$$

Using inequalities (2) and (3), it is enough to prove that

$$
\operatorname{depth} S /\left(I, x_{1} \ldots x_{d}\right) \geq \alpha_{2}(G)-s+1 \text {. }
$$


For every integer $k$ with $1 \leq k \leq d-1$, let $J_{k}$ be the ideal generated by all the squarefree monomials of degree $k$ on variables $x_{2}, \ldots, x_{d}$. We continue in the following steps.

Step 1. Let $1 \leq k \leq d-2$ be a fixed integer and assume that $\left\{u_{1}, \ldots, u_{t}\right\}$ is the set of minimal monomial generators of $x_{1} J_{k}$. In particular, every $u_{j}$ is divisible by $x_{1}$ and $\operatorname{deg}\left(u_{j}\right)=k+1$. For every integer $j$ with $1 \leq j \leq t$, we prove that

$$
\begin{aligned}
& \operatorname{depth} S /\left(I+x_{1} J_{k+1}+\left(u_{1}, \ldots, u_{j-1}\right)\right) \\
& \geq \min \left\{\operatorname{depth} S /\left(I+x_{1} J_{k+1}+\left(u_{1}, \ldots, u_{j}\right)\right), \alpha_{2}(G)-s+1\right\} .
\end{aligned}
$$

(Note that for $j=1$, we have $I+x_{1} J_{k+1}+\left(u_{1}, \ldots, u_{j-1}\right)=I+x_{1} J_{k+1}$.)

Consider the following short exact sequence.

$$
\begin{aligned}
& 0 \longrightarrow \frac{S}{\left(I+x_{1} J_{k+1}+\left(u_{1}, \ldots, u_{j-1}\right)\right): u_{j}} \longrightarrow \frac{S}{I+x_{1} J_{k+1}+\left(u_{1}, \ldots, u_{j-1}\right)} \\
& \longrightarrow \frac{S}{I+x_{1} J_{k+1}+\left(u_{1}, \ldots, u_{j}\right)} \longrightarrow 0
\end{aligned}
$$

As a consequence,

$\operatorname{depth} S /\left(I+x_{1} J_{k+1}+\left(u_{1}, \ldots, u_{j-1}\right)\right) \geq$

$\min \left\{\operatorname{depth} S /\left(\left(I+x_{1} J_{k+1}+\left(u_{1}, \ldots, u_{j-1}\right)\right): u_{j}\right), \operatorname{depth} S /\left(I+x_{1} J_{k+1}+\left(u_{1}, \ldots, u_{j}\right)\right)\right\}$.

Therefore, to complete this step, we need to show that

$$
\operatorname{depth} S /\left(\left(I+x_{1} J_{k+1}+\left(u_{1}, \ldots, u_{j-1}\right)\right): u_{j}\right) \geq \alpha_{2}(G)-s+1 .
$$

Set

$$
U_{j}:=\left\{x_{i} \mid 1 \leq i \leq d \text { and } x_{i} \text { does not divide } u_{j}\right\} .
$$

For any $x_{i} \in U_{j}$, the monomial $x_{i} u_{j}$ is a squarefree monomial of degree $k+2$. Hence, $x_{i} u_{j}$ belongs to $x_{1} J_{k+1}$. This shows that

(the ideal generated by $\left.U_{j}\right) \subseteq\left(\left(x_{1} J_{k+1}+\left(u_{1}, \ldots, u_{j-1}\right)\right): u_{j}\right)$.

We show the reverse inclusion holds too.

Since $x_{1} J_{k+1}+\left(u_{1}, \ldots, u_{j-1}\right)$ is a squarefree monomial ideal, it follows that

$$
\left(\left(x_{1} J_{k+1}+\left(u_{1}, \ldots, u_{j-1}\right)\right): u_{j}\right)
$$

is also a squarefree monomial ideal. On the other hand, $\left\{x_{1}, \ldots, x_{d}\right\}$ is the set of variables appearing in the set of minimal monomial generators of $x_{1} J_{k+1}+\left(u_{1}, \ldots, u_{j-1}\right)$. This implies that every monomial generator of $\left(\left(x_{1} J_{k+1}+\left(u_{1}, \ldots, u_{j-1}\right)\right): u_{j}\right)$ is a squarefree monomial over the variables $x_{1}, \ldots, x_{d}$. Assume that $v$ is a minimal generator of $\left(\left(x_{1} J_{k+1}+\left(u_{1}, \ldots, u_{j-1}\right)\right): u_{j}\right)$. If $v$ is not equal to any of the variables belonging to $U_{j}$, then by definition of $U_{j}$, every variable dividing $v$, also divides $u_{j}$. As $v$ is a squarefree monomial, we have $v \mid u_{j}$. Since

$$
u_{j} v \in x_{1} J_{k+1}+\left(u_{1}, \ldots, u_{j-1}\right),
$$


we deduce that

$$
u_{j}^{2} \in x_{1} J_{k+1}+\left(u_{1}, \ldots, u_{j-1}\right)
$$

which implies that

$$
u_{j} \in x_{1} J_{k+1}+\left(u_{1}, \ldots, u_{j-1}\right),
$$

because $x_{1} J_{k+1}+\left(u_{1}, \ldots, u_{j-1}\right)$ is a squarefree monomial ideal. This is contradiction, as the degree of $u_{j}$ is strictly less that the degree of any monomial in $x_{1} J_{k+1}$ and moreover none of the monomials $u_{1}, \ldots, u_{j-1}$ is equal to $u_{j}$. Hence,

$$
\left(\left(x_{1} J_{k+1}+\left(u_{1}, \ldots, u_{j-1}\right)\right): u_{j}\right)=\left(\text { the ideal generated by } U_{j}\right) .
$$

Let $W_{j}$ be the set of variables dividing $u_{j}$. In other words, $W_{j}=\left\{x_{1}, \ldots, x_{d}\right\} \backslash U_{j}$. We remind that for any pair of integers $1 \leq i, j \leq d$, the vertices $x_{i}$ and $x_{j}$ are not adjacent in $H^{\prime}$. Set

$$
U_{j}^{\prime}:=\bigcup_{x_{i} \in W_{j}} N_{H^{\prime}}\left[x_{i}\right]
$$

and

$$
U_{j}^{\prime \prime}:=\bigcup_{x_{i} \in W_{j}} N_{H^{\prime}}\left(x_{i}\right) .
$$

Using equality (4), we conclude that

$$
\begin{aligned}
& \left(\left(I+x_{1} J_{k+1}+\left(u_{1}, \ldots, u_{j-1}\right)\right): u_{j}\right)= \\
& \left(\left(I(H)^{(s)}+I\left(H^{\prime}\right)+x_{1} J_{k+1}+\left(u_{1}, \ldots, u_{j-1}\right)\right): u_{j}\right)= \\
& \left(I(H)^{(s)}: u_{j}\right)+I\left(H^{\prime} \backslash U_{j}^{\prime}\right)+\left(\text { the ideal generated by } U_{j} \cup U_{j}^{\prime \prime}\right) \\
& =\left(I\left(H \backslash\left(U_{j} \cup U_{j}^{\prime \prime}\right)\right)^{(s)}: u_{j}\right)+I\left(H^{\prime} \backslash\left(U_{j} \cup U_{j}^{\prime}\right)\right) \\
& +\left(\text { the ideal generated by } U_{j} \cup U_{j}^{\prime \prime}\right) .
\end{aligned}
$$

Set $H_{j}:=H \backslash\left(U_{j} \cup U_{j}^{\prime \prime}\right)$ and $H_{j}^{\prime}:=H^{\prime} \backslash\left(U_{j} \cup U_{j}^{\prime}\right)$. Then $H_{j}$ is a chordal graph, and $x_{1}$ is a simplicial vertex of $H_{j}$. It is also clear that $N_{H_{j}}\left[x_{1}\right]$ is the set of variables divining $u_{j}$. It thus follows from [14, Lemma 2] and the above equalities that

$$
\begin{aligned}
& \left(\left(I+x_{1} J_{k+1}+\left(u_{1}, \ldots, u_{j-1}\right)\right): u_{j}\right)=I\left(H_{j}\right)^{(s-k)}+I\left(H_{j}^{\prime}\right) \\
& +\left(\text { the ideal generated by } U_{j} \cup U_{j}^{\prime \prime}\right) .
\end{aligned}
$$

This yields that

$$
\operatorname{depth} S /\left(\left(I+x_{1} J_{k+1}+\left(u_{1}, \ldots, u_{j-1}\right)\right): u_{j}\right)=\operatorname{depth} S_{j} /\left(I\left(H_{j}\right)^{(s-k)}+I\left(H_{j}^{\prime}\right)\right),
$$

where $S_{j}=\mathbb{K}\left[x_{i}: 1 \leq i \leq n, i \notin U_{j} \cup U_{j}^{\prime \prime}\right]$. Let $G_{j}$ be the union of $H_{j}$ and $H_{j}^{\prime}$. Then $G_{j}$ is the induced subgraph of $G$ on $V(G) \backslash\left(U_{j} \cup U_{j}^{\prime \prime}\right)$. We conclude from Lemma 3.2 (by considering the clique $W_{j}$ ) that

$$
\alpha_{2}\left(G_{j}\right) \geq \alpha_{2}(G)-\left|W_{j}\right|+1=\alpha_{2}(G)-k,
$$


where the last equality follows from the fact that $\operatorname{deg}\left(u_{j}\right)=k+1$. Hence, the induction hypothesis implies that

$$
\begin{aligned}
& \operatorname{depth} S /\left(\left(I+x_{1} J_{k+1}+\left(u_{1}, \ldots, u_{j-1}\right)\right): u_{j}\right)=\operatorname{depth} S_{j} /\left(I\left(H_{j}\right)^{(s-k)}+I\left(H_{j}^{\prime}\right)\right) \\
& \geq \alpha_{2}\left(G_{j}\right)-(s-k)+1 \geq \alpha_{2}(G)-s+1,
\end{aligned}
$$

and this step is complete.

Step 2. Let $1 \leq k \leq d-2$ be a fixed integer. By a repeated use of Step 1 , we have $\operatorname{depth} S /\left(I+x_{1} J_{k+1}\right) \geq \min \left\{\operatorname{depth} S /\left(I+x_{1} J_{k+1}+\left(u_{1}, \ldots, u_{t}\right)\right), \alpha_{2}(G)-s+1\right\}$ $=\min \left\{\operatorname{depth} S /\left(I+x_{1} J_{k}\right), \alpha_{2}(G)-s+1\right\}$.

Step 3. It follows from Step 2 that

$$
\begin{aligned}
& \operatorname{depth} S /\left(I, x_{1} \ldots x_{d}\right)=\operatorname{depth} S /\left(I+x_{1} J_{d-1}\right) \\
& \geq \min \left\{\operatorname{depth} S /\left(I+x_{1} J_{d-2}\right), \alpha_{2}(G)-s+1\right\} \\
& \geq \min \left\{\operatorname{depth} S /\left(I+x_{1} J_{d-3}\right), \alpha_{2}(G)-s+1\right\} \\
& \geq \cdots \geq \min \left\{\operatorname{depth} S /\left(I+x_{1} J_{1}\right), \alpha_{2}(G)-s+1\right\} .
\end{aligned}
$$

In particular,

(5)

$$
\operatorname{depth} S /\left(I, x_{1} \ldots x_{d}\right) \geq \min \left\{\operatorname{depth} S /\left(I+\left(x_{1} x_{2}, x_{1} x_{3}, \ldots, x_{1} x_{d}\right)\right), \alpha_{2}(G)-s+1\right\} \text {. }
$$

Step 4. Let $L$ be the graph obtained from $H$, by deleting the edges $x_{1} x_{2}, \ldots, x_{1} x_{d}$. Then $L$ is the disjoint union of $H \backslash x_{1}$ and the isolated vertex $x_{1}$. In particular, $L$ is a chordal graph. Also, let $L^{\prime}$ be the graph obtained from $H^{\prime}$, by adding the edges $x_{1} x_{2}, \ldots, x_{1} x_{d}$. Then

$$
E(L) \cap E\left(L^{\prime}\right)=\emptyset \quad \text { and } \quad E(L) \cup E\left(L^{\prime}\right)=E(G) .
$$

It follows from [15, Lemma 3.2] and the induction hypothesis that

$$
\begin{aligned}
& \operatorname{depth} S /\left(I+\left(x_{1} x_{2}, x_{1} x_{3}, \ldots, x_{1} x_{d}\right)\right)= \\
& \operatorname{depth} S /\left(I(H)^{(s)}+I\left(H^{\prime}\right)+\left(x_{1} x_{2}, x_{1} x_{3}, \ldots, x_{1} x_{d}\right)\right)= \\
& \operatorname{depth} S /\left(\left(I(L)^{(s)}+I\left(L^{\prime}\right)\right)\right) \geq \alpha_{2}(G)-s+1 .
\end{aligned}
$$

Finally, inequality (5) implies that

$$
\operatorname{depth} S /\left(I, x_{1} \ldots x_{d}\right) \geq \alpha_{2}(G)-s+1 \text {. }
$$

Now, inequalities (2), (3) and (6) complete the proof of the proposition.

The following theorem is the main result of this section and follows easily from Proposition 3.3 .

Theorem 3.4. Let $G$ be a chordal graph. Then for every integer $s \geq 1$, we have

$$
\operatorname{depth} S /\left(I(G)^{(s)}\right) \geq \alpha_{2}(G)-s+1 .
$$


Proof. The assertion follows from Proposition 3.3 by substituting $H=G$ and $H^{\prime}=$ $\emptyset$.

\section{SECOND SYMBOLIC POWER OF EDGE IDEALS}

The aim of this section is to show that inequality $(\mp)$ is true for $s=2$, Theorem 4.2 . To prove this result, we need to bound the depth of ideals of the form $\left(I(G)^{(k)}: x y\right)$, where $x y$ is an edge of $G$. To achieve this goal, we will use the following lemma in the case of $k=2$.

Lemma 4.1. Let $G$ be a graph and $x y$ be an edge of $G$. Then for any integer $k \geq 2$, we have

$$
\left(I(G)^{(k)}: x y\right)=\left(I(G)^{(k-1)}: x\right) \cap\left(I(G)^{(k-1)}: y\right) .
$$

Proof. Let $u$ be a monomial in $\left(I(G)^{(k)}: x y\right)$. Then $u x y \in I(G)^{(k)}$. Clearly, this implies that $u x \in I(G)^{(k-1)}$. Therefore, $u \in\left(I(G)^{(k-1)}: x\right)$. Similarly, $u$ belongs to $\left(I(G)^{(k-1)}: y\right)$. Hence,

$$
\left(I(G)^{(k)}: x y\right) \subseteq\left(I(G)^{(k-1)}: x\right) \cap\left(I(G)^{(k-1)}: y\right) .
$$

To prove the reverse inclusion, let $v$ be a monomial in

$$
\left(I(G)^{(k-1)}: x\right) \cap\left(I(G)^{(k-1)}: y\right) .
$$

We must show that $v x y \in I(G)^{(k)}$. It is enough to prove that for any minimal vertex cover $C$ of $G$, we have $v x y \in \mathfrak{p}_{C}^{k}$. So, let $C$ be a minimal vertex cover of $G$. It follows from $x y \in E(G)$ that $C$ contains at least one of the vertices $x$ and $y$. Without lose of generality, suppose $x \in C$. Since $v \in\left(I(G)^{(k-1)}: y\right)$, we have $v y \in I(G)^{(k-1)} \subseteq \mathfrak{p}_{C}^{k-1}$. This together with $x \in \mathfrak{p}_{C}$ implies that $v x y \in \mathfrak{p}_{C}^{k}$.

The following theorem is the second main result of this paper.

Theorem 4.2. For any graph $G$, we have

$$
\operatorname{depth} S / I(G)^{(2)} \geq \alpha_{2}(G)-1 \text {. }
$$

Proof. Set $I:=I(G)$ and let $G(I)=\left\{u_{1}, \ldots, u_{m}\right\}$ be the set of minimal monomial generators of $I$. Using [1, Theorem 4.12], we may assume that for every pair of integers $1 \leq j<i \leq m$, one of the following conditions holds.

(i) $\left(u_{j}: u_{i}\right) \subseteq\left(I^{2}: u_{i}\right) \subseteq\left(I^{(2)}: u_{i}\right)$; or

(ii) there exists an integer $k \leq i-1$ such that $\left(u_{k}: u_{i}\right)$ is generated by a subset of variables, and $\left(u_{j}: u_{i}\right) \subseteq\left(u_{k}: u_{i}\right)$.

For every integer $i$ with $1 \leq i \leq m$ consider the short exact sequence

$$
\begin{aligned}
0 & \longrightarrow \frac{S}{\left(I^{(2)}+\left(u_{1}, \ldots, u_{i-1}\right)\right): u_{i}} \longrightarrow \frac{S}{I^{(2)}+\left(u_{1}, \ldots, u_{i-1}\right)} \\
& \longrightarrow \frac{S}{I^{(2)}+\left(u_{1}, \ldots, u_{i}\right)} \longrightarrow 0 .
\end{aligned}
$$


It follows from depth Lemma [2, Proposition 1.2.9] that

$$
\begin{aligned}
& \operatorname{depth} S /\left(I^{(2)}+\left(u_{1}, \ldots, u_{i-1}\right)\right) \\
& \geq \min \left\{\operatorname{depth} S /\left(\left(I^{(2)}+\left(u_{1}, \ldots, u_{i-1}\right)\right): u_{i}\right), \operatorname{depth} S /\left(I^{(2)}+\left(u_{1}, \ldots, u_{i}\right)\right)\right\} .
\end{aligned}
$$

Consequently,

$$
\begin{aligned}
& \operatorname{depth} S / I^{(2)} \geq \\
& \min \left\{\operatorname{depth} S /\left(I^{(2)}+I\right), \operatorname{depth} S /\left(\left(I^{(2)}+\left(u_{1}, \ldots, u_{i-1}\right)\right): u_{i}\right) \mid 1 \leq i \leq m\right\}= \\
& \min \left\{\operatorname{depth} S / I, \operatorname{depth} S /\left(\left(I^{(2)}+\left(u_{1}, \ldots, u_{i-1}\right)\right): u_{i}\right) \mid 1 \leq i \leq m\right\} \geq \\
& \min \left\{\alpha_{2}(G), \operatorname{depth} S /\left(\left(I^{(2)}+\left(u_{1}, \ldots, u_{i-1}\right)\right): u_{i}\right) \mid 1 \leq i \leq m\right\},
\end{aligned}
$$

where the last inequality follows from Corollary 2.2. Hence, it is enough to show that

$$
\operatorname{depth} S /\left(\left(I^{(2)}+\left(u_{1}, \ldots, u_{i-1}\right)\right): u_{i}\right) \geq \alpha_{2}(G)-1,
$$

for every integer $i$ with $1 \leq i \leq m$.

Fix an integer $i$ with $1 \leq i \leq m$ and assume that $u_{i}=x y$. We know from (i) and (ii) above that

$$
\left(\left(I^{(2)}, u_{1}, \ldots, u_{i-1}\right): u_{i}\right)=\left(I^{(2)}: u_{i}\right)+(\text { some variables }) .
$$

Let $A$ be the set of variables appearing in $\left(\left(I^{(2)}, u_{1}, \ldots, u_{i-1}\right): u_{i}\right)$. Assume that $x \in A$. This means that $x^{2} y$ belongs to the ideal $\left(I^{(2)}, u_{1}, \ldots, u_{i-1}\right)$. Since $u_{1}, \ldots, u_{i-1}$ do not divide $x^{2} y$, we deduce that $x^{2} y \in I(G)^{(2)}$. But this is a contradiction, as $C:=V(G) \backslash\{x\}$ is a vertex cover of $G$ with $x^{2} y \notin \mathfrak{p}_{C}^{2}$. Therefore, $x \notin A$. Similarly, $y \notin A$. It follows from $x, y \notin A$ and equality (17) that

$$
\begin{aligned}
& \left(\left(I^{(2)}, u_{1}, \ldots, u_{i-1}\right): u_{i}\right)=\left(I^{(2)}: u_{i}\right)+(\text { the ideal generated by } A) \\
& \left.=\left(I^{(2)}+(\text { the ideal generated by } A)\right): u_{i}\right) \\
& \left.=\left(I(G \backslash A)^{(2)}+(\text { the ideal generated by } A)\right): u_{i}\right) \\
& =\left(I(G \backslash A)^{(2)}: u_{i}\right)+(\text { the ideal generated by } A) .
\end{aligned}
$$

Therefore,

$$
\operatorname{depth} S /\left(\left(I^{(2)}, u_{1}, \ldots, u_{i-1}\right): u_{i}\right)=\operatorname{depth} S_{A} /\left(I(G \backslash A)^{(2)}: u_{i}\right),
$$

where $S_{A}=\mathbb{K}\left[x_{i}: 1 \leq i \leq n, x_{i} \notin A\right]$. It follows from Lemma 4.1 that

$$
\left(I(G \backslash A)^{(2)}: u_{i}\right)=(I(G \backslash A): x) \cap(I(G \backslash A): y) .
$$

Consider the following short exact sequence.

$$
\begin{aligned}
0 & \longrightarrow \frac{S_{A}}{\left(I(G \backslash A)^{(2)}: u_{i}\right)} \longrightarrow \frac{S_{A}}{(I(G \backslash A): x)} \oplus \frac{S_{A}}{(I(G \backslash A): y)} \\
& \longrightarrow \frac{S_{A}}{(I(G \backslash A): x)+(I(G \backslash A): y)} \longrightarrow 0
\end{aligned}
$$


Applying depth Lemma [2, Proposition 1.2.9] on the above exact sequence, it suffices to prove that

(a) $\operatorname{depth} S_{A} /(I(G \backslash A): x) \geq \alpha_{2}(G)-1$,

(b) depth $S_{A} /(I(G \backslash A): y) \geq \alpha_{2}(G)-1$, and

(c) $\operatorname{depth} S_{A} /((I(G \backslash A): x)+(I(G \backslash A): y)) \geq \alpha_{2}(G)-2$.

To prove (a), note that

$$
\begin{aligned}
& (I(G \backslash A): x)=I\left(G \backslash\left(A \cup N_{G \backslash A}[x]\right)\right)+\left(\text { the ideal generated by } N_{G \backslash A}(x)\right) \\
& =I\left(G \backslash\left(A \cup N_{G}[x]\right)\right)+\left(\text { the ideal generated by } N_{G \backslash A}(x)\right) .
\end{aligned}
$$

Hence,

$$
\operatorname{depth} S_{A} /(I(G \backslash A): x)=\operatorname{depth} S^{\prime} / I\left(G \backslash\left(A \cup N_{G}[x]\right)\right),
$$

where $S^{\prime}=\mathbb{K}\left[x_{i}: 1 \leq i \leq n, x_{i} \notin A \cup N_{G}(x)\right]$. Obviously, $x$ is a regular element of $S^{\prime} / I\left(G \backslash\left(A \cup N_{G}[x]\right)\right)$. Therefor, Corollary 2.2 implies that

$$
\operatorname{depth} S^{\prime} / I\left(G \backslash\left(A \cup N_{G}[x]\right)\right) \geq \alpha_{2}\left(G \backslash\left(A \cup N_{G}[x]\right)\right)+1 .
$$

Assume that $A \subseteq N_{G}(x) \cup N_{G}(y)$. It then follows from Lemma 3.1 that

$$
\alpha_{2}\left(G \backslash\left(A \cup N_{G}[x]\right)\right) \geq \alpha_{2}(G)-2 .
$$

Hence, we conclude from equality (9) and inequality (10) that

$$
\operatorname{depth} S_{A} /(I(G \backslash A): x) \geq \alpha_{2}(G)-1 .
$$

Thus, to complete the proof of (a), we only need to show that $A \subseteq N_{G}(x) \cup N_{G}(y)$.

Let $z$ be an arbitrary variable in $A$ and suppose $z \notin N_{G}(x) \cup N_{G}(y)$. Then the only edge dividing $z u_{i}=z x y$ is $u_{i}$. In particular,

$$
z \notin\left(\left(u_{1}, \ldots, u_{i-1}\right): u_{i}\right) .
$$

Moreover, since $\{z, x\}$ is an independent of subset of vertices of $G$, we conclude that $C^{\prime}=V(G) \backslash\{z, x\}$ is a vertex cover of $G$ with $z u_{i}=z x y \notin \mathfrak{p}_{C}^{2}$. Thus, $z u_{i} \notin I^{(2)}$. This means that $z \notin\left(I^{(2)}: u_{i}\right)$. This, together with (11) implies that

$$
z \notin\left(\left(I^{(2)}, u_{1}, \ldots, u_{i-1}\right): u_{i}\right)
$$

which is a contradiction. Therefore, $z \in N_{G}(x) \cup N_{G}(y)$. Hence, $A \subseteq N_{G}(x) \cup N_{G}(y)$ and this completes the proof of (a). The proof of (b) is similar to the proof of (a). We now prove (c).

Note that

$(I(G \backslash A): x)+(I(G \backslash A): y)=$

$I\left(G \backslash\left(A \cup N_{G \backslash A}[x] \cup N_{G \backslash A}[y]\right)\right)+\left(\right.$ the ideal generated by $\left.N_{G \backslash A}[x] \cup N_{G \backslash A}[y]\right)=$

$I\left(G \backslash\left(A \cup N_{G}[x] \cup N_{G}[y]\right)\right)+\left(\right.$ the ideal generated by $\left.N_{G \backslash A}[x] \cup N_{G \backslash A}[y]\right)=$

$I\left(G \backslash\left(N_{G}[x] \cup N_{G}[y]\right)\right)+\left(\right.$ the ideal generated by $\left.N_{G \backslash A}[x] \cup N_{G \backslash A}[y]\right)$, 
Where the last equality follows from $A \subseteq\left(N_{G}(x) \cup N_{G}(y)\right)$. We conclude that (12)

$$
\operatorname{depth} S_{A} /((I(G \backslash A): x)+(I(G \backslash A): y))=\operatorname{depth} S^{\prime \prime} / I\left(G \backslash\left(N_{G}[x] \cup N_{G}[y]\right)\right),
$$

where $S^{\prime \prime}=\mathbb{K}\left[x_{i}: 1 \leq i \leq n, x_{i} \notin N_{G}[x] \cup N_{G}[y]\right]$. Using Corollary 2.2 and Lemma 3.1, we deuce that

$$
\operatorname{depth} S^{\prime \prime} / I\left(G \backslash\left(N_{G}[x] \cup N_{G}[y]\right)\right) \geq \alpha_{2}\left(G \backslash\left(N_{G}[x] \cup N_{G}[y]\right)\right) \geq \alpha_{2}(G)-2 .
$$

Finally, the assertion of (c) follows from equality (12) and the above inequality. This completes the proof of the theorem.

\section{REFERENCES}

[1] A. Banerjee, The regularity of powers of edge ideals, J. Algebraic Combin. 41 (2015), 303-321.

[2] W. Bruns, J. Herzog, Cohen-Macaulay Rings, Cambridge Studies in Advanced Mathematics, 39, Cambridge University Press, 1993.

[3] L. Burch, Codimension and analytic spread, Proc. Cambridge Philos. Soc. 72 (1972), 369-373.

[4] G. Caviglia, H. T. Hà, J. Herzog, M. Kummini, N. Terai, N. V.Trung, Depth and regularity modulo a principal ideal, J. Algebraic Combin. 49 (2019), 1-20.

[5] H. Dao, J. Schweig, Bounding the projective dimension of a squarefree monomial ideal via domination in clutters, Proc. Amer. Math. Soc., 143 (2015), 555-565.

[6] L. Fouli, H. T. Hà, S. Morey, Depth of Powers of Squarefree Monomial Ideals, preprint.

[7] L. Fouli, H. T. Hà, S. Morey, Initially regular sequences and depth of ideals, preprint.

[8] L. Fouli, S. Morey, A lower bound for depths of powers of edge ideals, J. Algebraic Combin. 42 (2015), 829-848.

[9] H. T. Hà, N. V. Trung, T. N. Trung, Depth and regularity of powers of sums of ideals, Math. Z., 282 (2016), 819-838.

[10] J. Herzog, T. Hibi, Monomial Ideals, Springer-Verlag, 2011.

[11] J. Herzog, T. Hibi, The depth of powers of an ideal, J. Algebra 291 (2005), no. 2, 325-650.

[12] L. T. Hoa, K. Kimura, N. Terai, T. N. Trung, Stability of depths of symbolic powers of StanleyReisner ideal, J. Algebra 473 (2017), 307-323.

[13] H. D. Nguyen, N. V. Trung, Depth functions of symbolic powers of homogeneous ideals, Invent. Math, 218 (2019), 779-827.

[14] S. A. Seyed Fakhari, An upper bound for the regularity of symbolic powers of edge ideals of chordal graphs, Electron. J. Combin., 26 (2019), no. 2, Research Paper P2.10.

[15] S. A. Seyed Fakhari, Regularity of symbolic powers of edge ideals of unicyclic graphs, J. Algebra, 541 (2020), 345-358.

[16] A. Simis, W. Vasconcelos, R. H. Villarreal, On the ideal theory ofgraphs, J. Algebra 167 (1994), $389-416$.

S. A. Seyed Fakhari, School of Mathematics, Statistics and Computer Science, College of Science, University of Tehran, Tehran, Iran.

E-mail address: aminfakhari@ut.ac.ir 